OPEN ACCESS

Edited by: Anthony Christopher Vernon,

King's College London, United Kingdom

Reviewed by:

Gabriel R. Fries,

University of Texas Health Science Center at Houston, United States Caroline Menard,

Laval University, Canada

*Correspondence:

Cynthia Calkin

cindy.calkin@nshealth.ca

Specialty section:

This article was submitted to Schizophrenia,

a section of the journal

Frontiers in Psychiatry

Received: 01 December 2020 Accepted: 30 April 2021 Published: 25 May 2021

Citation:

Calkin C, McClelland C, Cairns K Kamintsky L and Friedman A (2021) Insulin Resistance and Blood-Brain

Barrier Dysfunction Underlie

Neuroprogression in Bipolar Disorder.

Front. Psychiatry 12:636174

doi: 10.3389/fpsyt.2021.636174

\section{Insulin Resistance and Blood-Brain Barrier Dysfunction Underlie Neuroprogression in Bipolar Disorder}

\author{
Cynthia Calkin ${ }^{1,2 *}$, Christie McClelland ${ }^{1}$, Kathleen Cairns ${ }^{3}$, Lyna Kamintsky ${ }^{2}$ and \\ Alon Friedman ${ }^{2,4}$
}

${ }^{1}$ Department of Psychiatry, Dalhousie University, Halifax, NS, Canada, ${ }^{2}$ Department of Medical Neuroscience, Dalhousie University, Halifax, NS, Canada, ${ }^{3}$ Nova Scotia Health, Halifax, NS, Canada, ${ }^{4}$ Departments of Cell Biology and Physiology, Ben-Gurion University of the Negev, Beer-Sheva, Israel

Bipolar disorder (BD) often progresses to a more chronic and treatment resistant (neuroprogressive) course. Identifying which patients are at risk could allow for early intervention and prevention. Bipolar disorder is highly comorbid with metabolic disorders including type II diabetes mellitus (T2DM), hypertension, obesity, and dyslipidemia. Our studies have shown that insulin resistance $(\mathrm{IR})$ is present in over $50 \%$ of patients with $\mathrm{BD}$ and that IR might underlie the progression of BD. While no confirmed predictors exist for identifying which patients with BD are likely to develop a more chronic course, emerging evidence including our own studies suggest that IR and related inflammatory pathways lead to impairments in blood-brain barrier (BBB) functioning. For the first time in living psychiatric patients, we have shown that the severity of BBB leakage is proportional to $\mathrm{BD}$ severity and is associated with IR. In this hypothesis paper we (i) highlight the evidence for a key role of $\mathrm{IR}$ in $\mathrm{BD}$, (ii) show how $\mathrm{IR}$ in $\mathrm{BD}$ relates to shared inflammatory pathways, and (iii) hypothesize that these modulations result in BBB leakage and worse outcomes in BD. We further hypothesize that (iv) reversing IR through lifestyle changes or the actions of insulin sensitizing medications such as metformin, or optimizing BBB function using vascular protective drugs, such as losartan, could provide novel strategies for the prevention or treatment of neuroprogressive BD.

Keywords: bipolar disorder, blood-brain barrier, insulin resistance, neuroprogression, vascular damage, inflammation

\section{INTRODUCTION}

Bipolar disorder is a mood disorder affecting up to $5 \%$ of the population, leading to significant morbidity and premature mortality. Mood dysregulation occurs in conjunction with symptoms affecting sleep, energy, interests/motivation, appetite/weight, concentration, speech, thought process, and judgment. Patients experience episodes of mania with or without depressive episodes in bipolar I disorder and recurrent episodes of hypomania and depression in bipolar II disorder. Treatment-resistant disease progression (neuroprogression) is not uncommon in bipolar patients and includes a shift toward more severe, prolonged and frequent mood episodes, including rapid cycling (a minimum of 4 discrete mood episodes yearly) (Figure 1A), and poor functional outcomes $(1,2)$. Until now there have been no validated predictors for which patients will progress to this advanced course and there are no corresponding treatments. Increasing evidence suggests 
that comorbid metabolic dysregulation, and specifically IR might underlie the progression of $\mathrm{BD}$ (3). We have found that those with comorbid T2DM or IR were more likely to develop a chronic course of $\mathrm{BD}$, more rapid cycling, and were less likely to respond to lithium compared to those without metabolic dysregulation (4). Similarly, poor outcomes in BD with comorbid IR have been reported, including worse cognitive decline (5), memory impairment (6), and poor response to mood stabilizers in general (7). Understanding mechanisms underlying these findings could lead to novel therapeutic or adjunctive treatment strategies.

Insulin resistance is an inflammatory state which affects the vasculature and can lead to endothelial changes in the BBB (8) (Figure 1B). We have recently examined the potential role of BBB dysfunction (BBBD) in BD using dynamic contrastenhanced magnetic resonance imaging (DCE-MRI). We found that $\mathrm{BD}$ patients with extensive $\mathrm{BBB}$ leakage had a more chronic course, greater severity of depression and anxiety, and poorer overall functioning compared with BD patients with normal BBB permeability (9). Further, we found that all patients with extensive BBB leakage also had IR. Expanding on our earlier hypothesis that IR plays a role in the development of neuroprogression in $\mathrm{BD}$ (3), we now propose that both IR and $\mathrm{BBBD}$ are biomarkers for neuroprogression and advancement of the illness process. We suggest that neuroprogression in patients with $\mathrm{BD}$ may be the result of comorbid IR and its effect on the integrity of the $\mathrm{BBB}$, as mediated through shared inflammatory pathways (Figure 1B).

\section{METABOLIC DYSREGULATION IN BD}

Patients with $\mathrm{BD}$ are symptomatic almost half of their lives (10). The leading cause of death in $\mathrm{BD}$ is cardiovascular disease with a 2.3-fold increased risk in patients with BD compared to the general population (11). Rates of cardiovascular risk factors including obesity, hypertension, dyslipidemia, metabolic syndrome and T2DM are all higher in patients with $\mathrm{BD}$ (12-14), and bipolar patients with comorbid T2DM have higher rates of hypertension, obesity, and a more chronic course (15). Obesity is a chronic, proinflammatory state, and adipose tissue secretes cytokines and inflammatory mediators (16) (Figures 1B,C) leading to further subsequent systemic inflammation and worsening obesity. This eventually results in an inadequate increase in insulin in response to plasma glucose, leading to IR with progression to glucose intolerance and eventually T2DM (17). We have reported that BD patients with comorbid obesity also have a more chronic course of illness and poor response to lithium (18). Similarly, worse outcomes in BD have been reported with comorbid IR, including worse cognitive decline (5).

Insulin resistance is present in more than half of all bipolar patients and is associated with a three times higher likelihood of a chronic course of illness with significantly more mood episodes including rapid cycling compared to those without metabolic dysregulation (4). These findings remained significant after controlling for the potential effects of age, body mass index (BMI), and lifetime exposure to antipsychotic medication
(4). This is important, given that many medications used to treat $\mathrm{BD}$ can also lead to the development of metabolic dysfunction (19), although the association between BD and metabolic dysregulation was described as early as 1921, well before the advent of psychotropic medications $(20,21)$. We have also reported that psychiatric morbidity in $\mathrm{BD}$ increases 12 fold following the onset of IR, and we have identified IR as an early manifestation or possible predictor of progression of BD (22).

Insulin resistance might also be useful in predicting poor response to treatment. In a 2015 study, we found that bipolar patients with either T2DM or IR were 8.4 times more likely to have a poor response to lithium (the gold-standard treatment) than bipolar patients with normal glucose metabolism (4). An inverse relationship between IR and response to lithium was demonstrated, such that as IR progressed, a poorer response to lithium was found (4). Similarly, Steardo et al. (7) found that bipolar patients with comorbid IR/T2DM were 4.3 times more likely to fail treatment with any mood stabilizer, including lithium. Furthermore, we found that IR preceded neuroprogression of $\mathrm{BD}$ (22), highlighting the potential to modify $\mathrm{BD}$ trajectory through early treatment of IR. Together, these findings stress the importance of monitoring IR in BD patients $(23,24)$ for early identification of neuroprogression and targeted treatment strategies. Targeting IR in bipolar patients may not only facilitate $\mathrm{BD}$ remission; but could also decrease the risk for T2DM, cardiovascular disease and dementia (3).

\section{HYPOTHALAMIC PITUITARY ADRENAL (HPA) DYSFUNCTION LEADS TO SYSTEMIC INFLAMMATION}

We identify the key role of the HPA axis in the development of both $\mathrm{BD}$ and IR (25-27), via induction of sustained hypercortisolemia (Figure 1B). Sustained hypercortisolemia causes the body to mount an immune response $(25,28)$, leading to systemic inflammation $(29,30)$, obesity, and increased risk of IR. Bipolar disorder is also associated with abnormalities of HPA axis activity, including increased levels of cortisol and adrenocorticotropic hormone (ACTH) $(26,27)$ along with disruption in the normal diurnal variation of cortisol. Specifically there is an absence of expected cortisol troughs at night (31) and higher than usual daytime elevations (32), contributing to circadian rhythm dysfunction. Bipolar patients also have altered sleep and cortisol levels even when euthymic (33) and poor sleep initiation and frequent nighttime awakenings increase risk for IR/T2DM (34).

\section{SYSTEMIC INFLAMMATION LEADS TO NEUROINFLAMMATION AND NEUROPROGRESSION}

We further propose that systemic inflammation and IR increase the risk of endothelial injury, dysfunction of the $\mathrm{BBB}$ and subsequent neuroinflammation (8) (Figure 1B). 


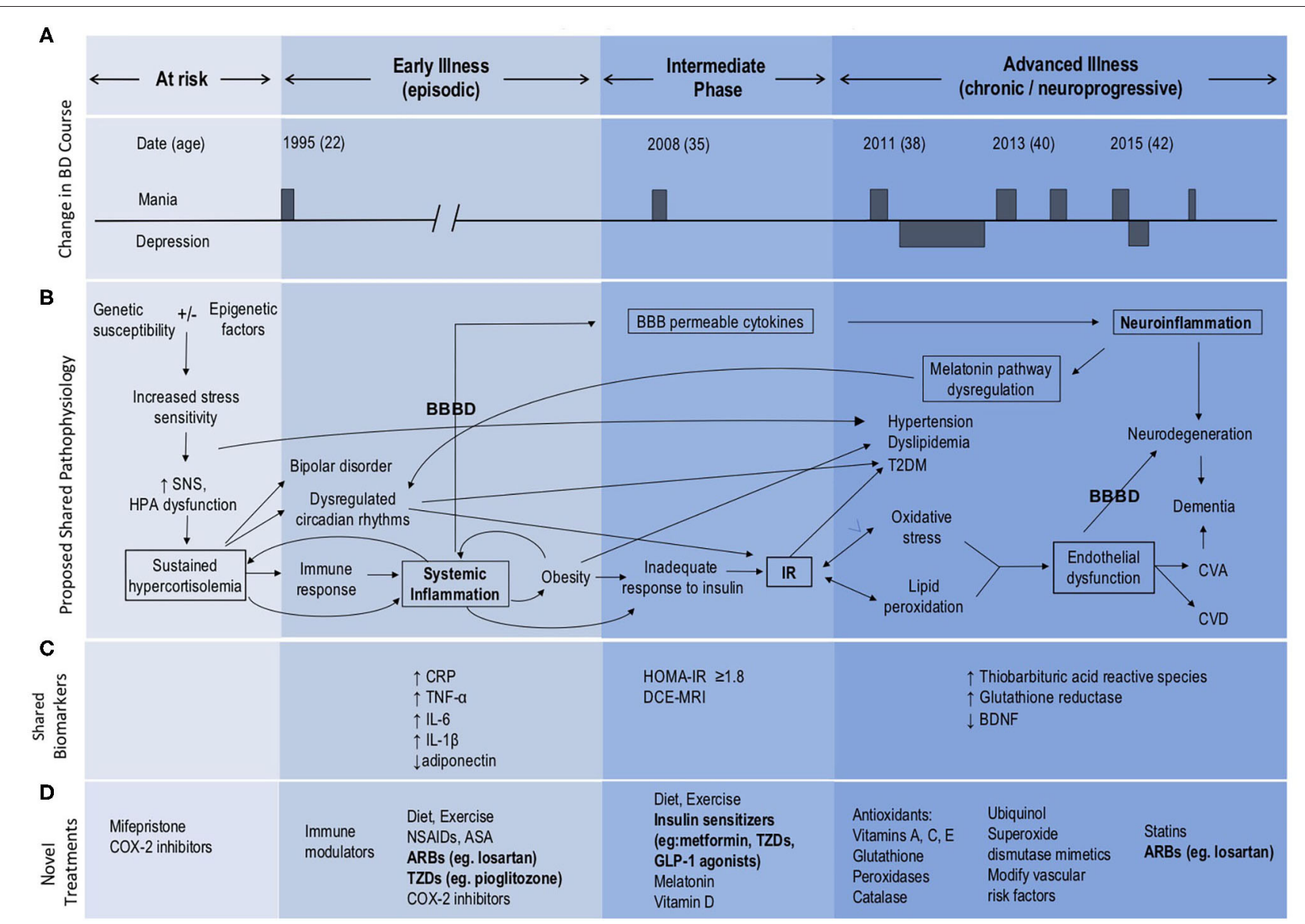

FIGURE 1 | Model of BD neuroprogression mediated by IR and BBBD. ARBs, Angiotensin II receptor blockers; ASA, acetylsalicylic acid; BBB, blood-brain barrier; BBBD, blood-brain barrier dysfunction; BDNF, brain-derived neurotrophic factor; CNS, central nervous system; COX-2 inhibitors, Cyclooxygenase-2 inhibitors; CRP, C-reactive protein; CVA, cerebrovascular accident; CVD, cardiovascular disease; GLP, glucagon-like peptide; HOMA-IR, Homeostatic Model Assessment-Insulin Resistance; HPA, hypothalamic pituitary adrenal; IL, interleukin; IR, insulin resistance; NSAIDs, Non-steroidal anti-inflammatory drugs; SNS, sympathetic nervous system; TNF, tumor necrosis factor; T2DM, type 2 diabetes mellitus; TZDs, thiazolidinediones. This figure has been modified from the original published figure by Calkin C V., Insulin resistance takes center stage: a new paradigm in the progression of bipolar disorder, published in Annals of Medicine. Taylor and Francis Ltd; 2019.

Neuroinflammation then further amplifies BBBD, creating a self-reinforcing positive feedback loop that exacerbates BD, and contributes to its progression. In addition, hyperglycemia and hyperinsulinemia activate the renin-angiotensin system (RAS) which contributes to the development of hypertension and endothelial dysfunction Endothelial dysfunction results in microvascular and macrovascular changes, which when occurring within the brain lead to impairments in BBB functions. Given that even mild hyperglycemia can lead to profound dysfunction of the BBB (8), it is possible that early intervention with lifestyle and dietary changes (35) at the IR stage prior to the development of hyperglycemia could prevent or mitigate these effects on the BBB and development of neuroprogression. Further, targeting factors such as hypercortisolemia, sleep disturbances, IR, BBBD and/or neuroinflammation may offer novel therapeutic avenues for the management of $\mathrm{BD}$ and in preventing neuroprogression (Figure 1D).

\section{SYSTEMIC INFLAMMATION IN BD AND IR}

Mood disorders are understood to develop from a combination of genetic and environmental factors, which ultimately lead to a broad spectrum of clinical presentations. While the pathophysiology of these processes remains largely elusive, increasing evidence supports a key role of inflammatory cascades in $\operatorname{BD}(36,37)$. Inflammation is defined as a non-specific state, known to be caused by both internal and external factors and may represent the body's response as a defense to a perceived threat $(25,37)$. Patients with systemic autoimmune diseases have an increased propensity for the development of $\mathrm{BD}$, and several increased peripheral proinflammatory mediators have been reported in BD (38). Further, markers of neuroinflammation are present in the cerebrospinal fluid of living patients with BD (39), and post-mortem studies have demonstrated increased inflammatory markers in the 
frontal cortex (40). Along with BD, it is generally accepted that a mild inflammatory state occurs in major depressive disorder (MDD) and schizophrenia (41). Long-term exposure to cytokines can lead to depressive episodes in euthymic patients receiving immune therapy with INF-alpha (42-44). Similarly, the administration of pro-inflammatory cytokines may lead to depressive symptoms in healthy controls (45-47). Blockade of TNF-type cytokines in depressed subjects with comorbid diseases including rheumatoid arthritis, psoriasis, and cancer was found to significantly reduce depressive symptoms (48, 49). Additionally, improvement in psychiatric symptoms has been demonstrated in schizophrenia patients treated with antiinflammatory drugs for other indications (50).

The most prominent cytokines associated with mood disorders include: interleukin-1 beta (IL-1ß), interleukin-6 (IL-6), tumor necrosis factor alpha (TNF-alpha), and Creactive protein (CRP) (51) (see Figure 1C). Interleukin-6 was shown to be increased in $\mathrm{BD}$ patients when unwell. Interestingly, levels of IL-6 were decreased following 6 weeks of mood stabilizing treatment (52). Indeed, increased activity of pro-inflammatory cytokines and an imbalance with antiinflammatory cytokines have been implicated in the development of BD and neuroprogression (51-53) and (see Figure 1C). Of particular interest, varying cytokine profiles have been identified in distinct mood states of $\mathrm{BD}$, including in mania, depression, euthymia and in healthy controls $(54,55)$. Specifically, manic episodes are associated with a prominent pro-inflammatory profile state (38). It has also been shown that inflammatory markers positively correlate with symptom severity in BD (29, $56,57)$. In late-stage BD more extreme elevations in these serum markers have been found, especially TNF-alpha. As underscored by Benedetti et al. (58), increased inflammation has been linked to other known hallmarks of $\mathrm{BD}$, including white matter changes and structural alterations in the prefrontal cortex, hippocampus, and amygdala $(59,60)$.

Practically, inflammatory profiles could prove to be useful in predicting treatment response to antidepressants in depressed states. The presence of neuroinflammation has been shown to result in a decreased response to some antidepressants (61). Specifically, in a systematic review, the presence of an inflammatory state (raised serum CRP and IL-6) in MDD patients correlated with poor outcome and poor response to predominantly serotonergic antidepressants (61). A better response to antidepressant regimes was demonstrated with add-on noradrenergic, dopaminergic, or glutamatergic action (61). In rats with a depression-like phenotype, augmentation with acetylsalicylic acid (ASA), a non-selective cyclo-oxygenase (COX) inhibitor and anti-inflammatory, enhanced the efficacy of fluoxetine (62). Levels of immunological markers could also help to predict the efficacy of some medications in $\mathrm{BD}$, such as lithium response $(29,63-65)$. Overall evidence suggests that an activated inflammatory response is associated with treatment resistance in general and is possibly indicative of a different (i.e., neuroprogressive) disease phase. This draws a similarity to our findings of treatment resistance in bipolar patients with comorbid IR and thus, possibly, indicates a shared inflammatory mechanism.
Over years of research, various signaling pathways have been associated with mood disorders, including alterations in 5hydroxytryptamine (5-HT) receptor functioning, neurotrophins, and the HPA axis $(25,28,30)$. Immune dysregulation has also become a focused area of this research. However, the general framework regarded immune alterations as an association or consequence of mood disorders, as opposed to a causative factor. A growing body of research has questioned this framework. Indeed, genetic studies have shown that immune alterations are detectable even before the onset of BD (58). Moreover, inflammatory serum markers are also elevated in adolescents with $\mathrm{BD}$ prior to the development of a clear illness course (66). A recent systematic review by Mucci et al. (51) highlights inflammatory processes as the mechanism contributing to the onset of mood disorders following a stressful stimuli.

It has also been found that an increased production of cortisol in Cushing's disease leads to depressive and manic symptoms and neurocognitive deficits, and affects various central nervous system (CNS) regions (67). In addition to other commonalities, increased oxidative stress has been identified in both BD and T2DM. Insulin resistance initially stimulates an increase in metabolic activity, resulting in elevated production of reactive oxygen species (ROS), and a subsequent increase in inflammatory cytokines, such as TNF-alpha, Interleukin Beta (IL-ß), and monocyte chemoattractant protein-1 (MCP-1) (68). Importantly, oxidative stress markers such as nitric oxide (NO) and ROS have also been suggested to play a role in the pathophysiology of $\mathrm{BD}$ (57). Since oxidative stress and inflammation may contribute to both $\mathrm{BD}$ and IR, IR-related inflammation could underlie and precede $\mathrm{BD}$ neuroprogression, triggering a transition into a more chronic and treatment-resistant course of illness $(3,69$, 70) (see Figures 1A,B). Utilizing a growing body of research, we further propose that alterations in BBB functions play a critical role in this pathogenic cascade. Specifically, we suggest that: (i) IR and associated inflammation underlie microvascular injury and BBB dysfunction; and (ii) that high $\mathrm{BBB}$ permeability to serum components facilitates further neuroinflammation and dysfunction of the neurovascular unit-including neuronal networks, that underlie the chronic and neuroprogressive course of BD.

\section{BLOOD-BRAIN BARRIER DYSFUNCTION IN BD}

The $\mathrm{BBB}$ is a complex structural and functional interface tightly regulating molecular exchange between the circulation and brain tissue. The BBB is formed by endothelial cells, linked together by tight junction proteins, and surrounded by pericytes, and astroglial foot processes $(71,72)$. This complex structure restricts harmful molecules in the blood from entering the brain, while facilitating the entry of essential nutrients and removal of waste products $(73,74)$. The BBB is integral to healthy brain functioning, and pathological BBB has been associated with autoimmune diseases (75) and common neurological conditions (76-79). Increase in BBB permeability can be inferred indirectly, by measuring whether a patient's 
CSF has a high concentration of molecules that are normally excluded from the brain, for example, albumin or urate (80). High levels of these BBBD markers have been associated with MDD in elderly women, as well as suicidality in unipolar MDD patients (81-83) - supporting a possible role for BBBD in mood disorders. Post-mortem evidence of BBBD has been demonstrated in neuropsychiatric disorders such as dementia (84) and depression (85). Recent advances in MRI techniques have allowed direct assessment of $\mathrm{BBB}$ functionality in living patients, demonstrating BBBD in pathological conditions such as traumatic and ischemic injuries $(86,87)$, epilepsy $(77)$, dementia (78) multiple sclerosis (79), and systemic lupus erythematosus (SLE) (75).

The understanding of the molecular mechanisms involved in BBBD had been largely derived from experimental animal models. This growing body of evidence suggests that: (i) systemic inflammation is associated with injury to brain (and other organs) microvasculature, resulting in BBBD (88), and (ii) BBBD allows leakage of serum macromolecules into the brain, resulting in glial activation, neuro-inflammation, network reorganization and delayed neurodegeneration as well as further $\operatorname{BBBD}(71,73,89)$.

One widely studied example of a serum macromolecule that leaks into the brain following BBB dysfunction is albumin (80)the most abundant protein in the blood. Once leaking into the brain, albumin has been shown to trigger glial transformation, by activation of the pro-inflammatory transforming growth factor beta (TGF- $ß)$ pathway $(80,89,90)$, leading to cytokine secretion, synaptogenesis and neurodegeneration $(77,87,88)$. In mouse models of social defeat, stress was also linked to increased BBB permeability and subsequent leakage of molecules into the brain (91). Specifically, the tight junction protein claudin-5 was downregulated and promoted peripheral IL- 6 passage across the BBB (91). Claudin-5 expression was similarly downregulated in depressed patients (92). Using immunohistochemistry and qRTPCR, (92) a key role for claudin-5 which was reduced in the hippocampus in post-mortem human brain tissues in people diagnosed with MDD or schizophrenia was also demonstrated. Interestingly, levels of claudins including expression of claudin-5 correlated with disease duration and age of psychiatric disorder in these post-mortem studies (92). Indeed, BBB associated tight junction disruption could be a major step in the development of various psychiatric pathologies. This is further supported by post-mortem analysis of patients with $\mathrm{MDD}$, showing reduced astrocytic coverage of the $\mathrm{BBB}$ in the orbitofrontal cortex (85). Notably, post-mortem studies in human patients with BD have also demonstrated neuroinflammatory changes, microglial activation, and oligodendrocyte dysfunction (93). To add to the animal and post-mortem studies linking $\mathrm{BD}$, neuroinflammation, and BBBD, our group has recently conducted the first ever BBB imaging study in living bipolar patients (9). Using DCE-MRI, we have demonstrated that extensive BBB leakage affects $28 \%$ of BD patients (9), and that these patients had greater psychiatric morbidity, compared to $\mathrm{BD}$ patients with normal BBB function. Extensive BBB leakage was found to be associated with worse depression, anxiety and socio/occupational dysfunction, chronic illness course with more frequent and/or severe manic/depressive episodes (9). Importantly, all bipolar patients with extensive BBB leakage also had IR, supporting the hypothesis that IR-related inflammation may contribute to BBBD and BD progression. Our results suggest $\mathrm{BBBD}$ could indeed be a mechanistic link between systemic inflammation, IR and BD neuroprogression. Moreover, repair of the $\mathrm{BBB}$ may, thus, prove to be a novel and effective approach for maintaining brain health and facilitating $\mathrm{BD}$ remission.

\section{IR AND BLOOD-BRAIN BARRIER DYSFUNCTION}

Our finding that all BD patients with extensive BBB leakage also had IR (9), highlights a potential causal link between IR and BBBD. While animal and human studies suggest that inflammation is the likely mechanism connecting IR and BBBD, the direct mechanistic link between IR and BBBD in BD patients has yet to be studied. Evidence for the role of IR in BBBD can be found in animal studies of hyperglycemia, showing that hyperglycemia causes inflammation and damage to the BBB (8). Notably, numerous overlapping pathways are involved in the inflammatory states of both IR and BBBD, including vascular endothelial growth factor (VEGF) and protein kinase $\mathrm{C}$ (PKC) (94). Further evidence supporting a possible link between BBBD and IR comes from studies of $\mathrm{BBBD}$ in T2DM. Impairment of the $\mathrm{BBB}$ is now accepted as one of the key mechanisms leading to diabetic encephalopathy (95). The structural integrity and transport function of the BBB is compromised in T2DM, through pathways of oxidative stress and chronic inflammation (95). BBBD in T2DM has also been suggested to play a role in neuronal dysfunction (96), and cognitive impairment (95). Further, both human and animal studies have demonstrated a role for insulin in synaptic viability, dendritic spine formation, cerebral bioenergetics and suggest that insulin dysregulation can lead to diseases of pathological aging (97). Specifically, a relationship between IR in the periphery and the brain in Alzheimer's disease suggests that peripheral IR might precede accumulation of amyloid ß protein in Alzheimer's (97). Applying this concept to the current body of evidence in IR and $\mathrm{BD}$, we postulate that $\mathrm{BBBD}$ is likely to be a key mechanism contributing to $\mathrm{BD}$ neuroprogression, as mediated by IR and shared inflammatory pathways (Figure 1B).

\section{INTRACELLULAR MECHANISMS SUPPORTING A LINK BETWEEN IR, INFLAMMATION, BBBD, AND NEUROPROGRESSION}

In this paper we present a framework for development of $\mathrm{BD}$ neuroprogression (Figure 1B), centered around the transition from systemic inflammation to neuroinflammation via the BBB. We identify HPA dysfunction as an early stage of a pathological cascade that leads to sustained hypercortisolemia and circadian dysregulation that may underlie the parallel development of 


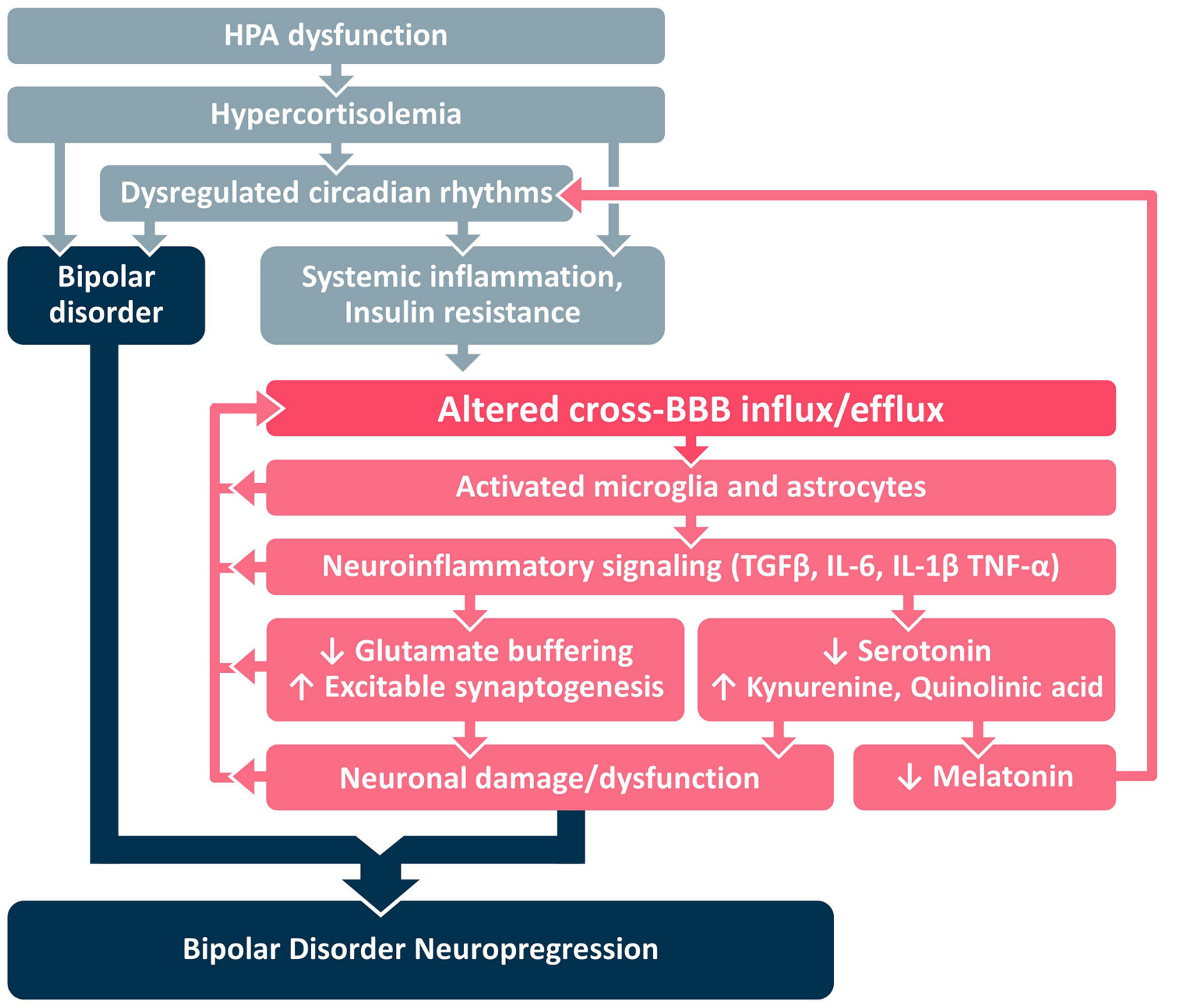

FIGURE 2 | Proposed mechanistic framework. BBB, blood-brain barrier; HPA, hypothalamic pituitary adrenal; IL, interleukin; TGFB, Transforming Growth Factor Beta; TNF, tumor necrosis factor.

both (a) BD and (b) systemic inflammation and IR (Figure 2). Systemic inflammation and IR leads to neuroinflammation via cross-BBB infiltration of systemically-secreted cytokines (e.g., IL1, IL-6, or TNF- $\alpha$ ) and/or damage to the BBB's endothelium mediated by hyperinsulinemia/hyperglycemia ( 8 , $17,98,99)$. Once the BBB is breached, the brain's microglial and astrocytic cells undergo a neuroinflammatory transformation, involving TGF $\beta$ pathway activation and further cytokine secretion. The astrocytic TGF $\beta$ cascade results in reduced buffering of extracellular glutamate (100), and generation of new excitatory synapses (91). Together these changes result in a reorganization of the neural network, a shift favoring hyperexcitation, and glutamate-mediated neuronal damage (101). Notably, each step of the neuroinflammatory cascade can contribute to further $\mathrm{BBBD}$, creating a self-reinforcing positive feedback loop that may also amplify the subsequent neuronal dysfunction. We hypothesize that the processes mediated by BBBD and neuroinflammation may impair the function of the affected brain regions, and contribute to the severity of $\mathrm{BD}$ and its responsiveness to available mood-stabilizing treatments. Interestingly, neuroinflammatory signaling cascades may also amplify the circadian dysregulation in BD patients, by favoring the production of kynurenine (and quinolinic acid) over serotonin (and melatonin) (102-104). Together, these pathways demonstrate the tight interplay between HPA activity, systemic inflammation, BBBD, neuroinflammation and $\mathrm{BD}$ neuroprogression, with the $\mathrm{BBB}$ being the interface between systemic and CNS processes/manifestations. 


\section{CAN TREATING IR AND/OR REPAIRING BBBD HALT BD NEUROPROGRESSION?}

Thus far, we have reviewed evidence suggesting that: (i) BBBD in bipolar patients might be caused by inflammatory processes such as IR, and (ii) BBBD may contribute to BD neuroprogression, that is more common in bipolar patients with IR. Research into manipulation of the BBB is becoming an area of growing interest, with several groups developing therapeutics targeting the repair of the $\mathrm{BBB}$ and/or inhibition of neuroinflammatory processes subsequent to BBBD (105-107). Given that BBBD in bipolar patients may be caused by systemic inflammation and IR, the question follows whether reversal of inflammation or IR could facilitate BBB repair and the remission of neuroprogression.

While some studies suggest that anti-inflammatory treatment or TNF inhibitors may reduce depressive symptoms $(48,49)$, whether they can repair the BBB remains unclear. A study in patients undergoing cardiac surgery suggests that treatment with the potent anti-inflammatory prednisone does not reduce post-operative BBBD (108). While this is perhaps far removed from $\mathrm{BD}$, conceptually, this suggests that treating systemic inflammation alone may not be sufficient to repair the BBB.

Studies in animals suggest that reversal of IR may be a more promising therapeutic target for BBB repair. Specifically, metformin-regarded as the safest and most effective insulinsensitizing drug-has been reported to reduce BBBD in a mouse model of stroke (109). In a mouse model of systemic inflammation using peripheral injection of bacterial lipopolysaccharide (LPS), metformin was shown to downregulate neuroinflammation and improve exploratory behavior (110). In this model, LPS was shown to cause neuroinflammation (elevated levels of TNF- $\alpha$ and IL- 6 in the brain), BBBD and significant lethargy and illness $(88,110)$. Remarkably, pre-treatment of mice with metformin significantly reduced systemic and CNS inflammation, LPS-induced oxidative stress, and neurobehavioral symptoms of illness (110). In humans, metformin has also been shown to enact antioxidant and anti-inflammatory effects, and to reduce cardiovascular complications, stroke, certain cancers, thyroid diseases, and polycystic ovarian syndrome (111-113).

Metformin is generally understood to exert its effects on the liver and peripheral tissues, by decreasing glucose output from the liver and increasing glucose utilization at peripheral tissues, including the musculature. This process requires the activation of adenosine monophosphate-activated protein kinase (AMPK) which reduces energy expenditure at the cellular level (112). However, metformin may offer further benefits beyond reversing IR. Metformin is also thought to suppresses the action of matrix metalloproteinase-9 (MMP9)-an enzyme that degrades components of the BBB (114). MMP-9 has been widely implicated in conditions such as cancer, MS, migraines, neuropsychiatric disorders $(115,116)$, and complications of coronary artery disease (116), atherosclerosis (117), and hypertension (118). Furthermore, increased levels of MMP-9 were demonstrated in bipolar depression (119), suggesting that MMP-9 could contribute to BBB degradation and disease progression of bipolar patients. Metformin has been shown to suppress MMP9 in human breast cancer cell lines, and is gaining focus as a potential anticancer drug (120).

An additional mechanism by which metformin may exert its neuroprotective effect is through activation of the peroxisome proliferator-activated receptor (PPAR) (121). PPAR is thought to mediate the insulin-sensitizing action of metformin, by modulating the insulin-like growth-factor (IGF) axis (121). In animal studies, PPAR agonists (thiazoladinediones, such as rosiglitosone) have been shown to protect the $\mathrm{BBB}$; reduce neuroinflammation, oxidative stress and neuronal injury $(120,122)$; and improve neurological outcomes of CNS injury/disease (123). Elegantly, PPAR- $\gamma$ antagonists lead to an opposite effect (122). These findings were further confirmed in a monolayer of human microvascular endothelial cells (122), suggesting that activation of PPAR signaling may be a promising neuroprotective target, and further highlighting the therapeutic potential of metformin and the thiazolidinedione class of drugs. Targeting the $\mathrm{BBB}$ directly, may indeed provide a promising approach in other CNS diseases, such as epilepsy which is also associated with BBBD (77). The angiotensin II receptor antagonist, losartan is vascular protective and has potential implications for protecting BBB integrity though action on TGF- 3 . In a rat model of vascular injury, losartan prevented acquired epilepsy via TGF$ß$ signaling suppression when administered prior to injury and may become the first available treatment for the prevention of epilepsy (105).

We conclude that treatment of IR and potential repair of the BBB with metformin, thiazolidinediones or vascular protective drugs, such as losartan may prove to be effective strategies for treatment resistant or neuroprogressive bipolar disorder (Figure 1D). Further research is needed to investigate the mechanisms underlying the effects of these agents on MMP9 and PPAR pathways in particular. By more directly targeting the $\mathrm{BBB}$, a shift in focus toward prevention of $\mathrm{BD}$ neuroprogression could emerge.

\section{EVALUATING IR AND BBBD TO IDENTIFY BD PATIENTS WITH NEUROPROGRESSION}

Insulin resistance can be easily estimated using concurrent FPG and FSI levels and the HOMA-IR equation: HOMA-IR = FPG $(\mathrm{mmol} / \mathrm{L}) \times$ FSI $(\mu \mathrm{U} / \mathrm{ml}) / 22.5(124)$, and the HOMA-IR cutoff value of $\geq 1.8$ (since metabolic syndrome becomes clinically significant at this value) (125). Once it is determined that a patient is insulin resistant, DCE-MRI could be used to quantify and localize BBB leakage. While DCE-MRI is clinically available and there are algorithms to analyze and interpret images for $\mathrm{BBBD}$, this has only been used for research. Standardizing abnormal BBB permeability cut-off values between different types of MRI scanners is required and feasible, but calls for large sample sizes, controls and further validation before it can be brought widely into clinical use. As our group is currently doing this work, we expect $\mathrm{BBB}$ imaging to be available for clinical use 
in the future. We suggest that utilizing these two biomarkers (IR, and BBBD once clinically available) will be important in identifying bipolar patients with neuroprogression and for following treatment response.

\section{DISCUSSION}

We have highlighted the evidence for a key role for IR and shared inflammatory pathways leading to BBBD and neuroprogression of $\mathrm{BD}$. We have outlined the rationale for our expanded hypothesis that these modulations result in $\mathrm{BBB}$ leakage and worse outcomes in $\mathrm{BD}$. We have further connected this body of evidence with key known treatments for IR. Indeed, reversing IR could provide a novel strategy for the prevention or treatment of a neuroprogressive course of $\mathrm{BD}$. This could be accomplished through the actions of widely used diabetic medications, such as metformin or thiazolidinediones or vascular protective agents, like losartan, that target BBBD more directly. While there is currently a lack of evidence to clearly determine whether treating IR or BBBD is of value in $\mathrm{BD}$ neuroprogression, our group has a number of studies under way. We are hopeful that data from our completed TRIO-BD quadruple-masked randomized clinical trial comparing metformin to placebo in improving outcomes in treatment resistant bipolar depression will soon help provide answers. Further research is needed in terms of replicating completed studies and investigating novel new treatments for $\mathrm{BD}$ neuroprogression, targeting IR and BBBD.

\section{REFERENCES}

1. Passos IC, Mwangi B, Vieta E, Berk M, Kapczinski F. Areas of controversy in neuroprogression in bipolar disorder. Acta Psychiatr Scand. (2016) 134:91103. doi: 10.1111/acps.12581

2. Fries GR, Pfaffenseller B, Stertz L, Paz AVC, Dargél AA, Kunz M, et al. Staging and neuroprogression in bipolar disorder. Curr Psychiatry Rep. (2012) 14:667-75. doi: 10.1007/s11920-012-0319-2

3. Calkin CV. Insulin Resistance Takes Center Stage: A New Paradigm in the Progression of Bipolar Disorder. Ann Med. (2019) 51:28193. doi: 10.1080/07853890.2019.1659511

4. Calkin CV, Ruzickova M, Uher R, Hajek T, Slaney CM, Garnham JS, et al. Insulin resistance and outcome in bipolar disorder. Br J Psychiatry. (2015) 206:52-7. doi: 10.1192/bjp.bp.114.152850

5. Cuperfain AB, Kennedy JL, Gonçalves VF. Overlapping mechanisms linking insulin resistance with cognition and neuroprogression in bipolar disorder. Neurosci Biobehav Rev. (2020) 111:12534. doi: 10.1016/j.neubiorev.2020.01.022

6. Salvi V, Salvo G Di, Korčáková J, Torriero S, Aragno E, Kolenič M, et al. Insulin resistance is associated with verbal memory impairment in bipolar disorders. J Affect Disord. (2020) 266:610-4. doi: 10.1016/j.jad.2020.01.145

7. Steardo L, Fabrazzo M, Sampogna G, Monteleone AM, D’Agostino G, Monteleone $\mathrm{P}$, et al. Impaired glucose metabolism in bipolar patients and response to mood stabilizer treatments. J Affect Disord. (2019) 245:1749. doi: 10.1016/j.jad.2018.10.360

8. Ennis SR, Keep RF. Effect of sustained-mild and transient-severe hyperglycemia on ischemia-induced blood-brain barrier opening. J Cereb Blood Flow Metab. (2007) 27:1573-82. doi: 10.1038/sj.jcbfm.9600454

9. Kamintsky L, Cairns KA, Veksler R, Bowen C, Beyea SD, Friedman A, et al. Blood-brain barrier imaging as a potential

\section{DATA AVAILABILITY STATEMENT}

The original contributions presented in the study are included in the article/supplementary material, further inquiries can be directed to the corresponding author/s.

\section{AUTHOR CONTRIBUTIONS}

CC contributed intellectual concepts, hypotheses, writing, and editing. CM synthesized these concepts and hypotheses in the writing of the manuscript and provided the literature search. $\mathrm{LK}, \mathrm{KC}$, and AF contributed to intellectual content, writing, and editing. All authors contributed to the article and approved the submitted version.

\section{FUNDING}

CC would like to thank the Brain \& Behavior Research Foundation (NARSAD, grant \# 18764 and grant \# 25714), and the Stanley Medical Research Institute (grant \# 14T-008) for awards supporting her research and AF would like to thank the Canadian Institutes for Health Research (grant \# PJT 148896) for supporting his research.

\section{ACKNOWLEDGMENTS}

We would like to thank Claire Reardon for help in preparing and formatting the manuscript.

biomarker for bipolar disorder progression. NeuroImage Clin. (2019) 26:102049. doi: 10.1016/j.nicl.2019.102049

10. Judd LL, Akiskal HS, Schettler PJ, Endicott J, Maser J, Solomon $\mathrm{DA}$, et al. The long-term natural history of the weekly symptomatic status of bipolar I disorder. Arch Gen Psychiatry. (2002) 59:5307. doi: 10.1001/archpsyc.59.6.530

11. Ösby U, Brandt L, Correia N, Ekbom A, Sparén P. Excess mortality in bipolar and unipolar disorder in Sweden. Arch Gen Psychiatry. (2001) 58:844-50. doi: 10.1001/archpsyc.58.9.844

12. Fagiolini A, Frank E, Scott JA, Turkin S, Kupfer DJ. Metabolic syndrome in bipolar disorder: findings from the bipolar disorder center for Pennsylvanians. Bipolar Disord. (2005) 7:424-30. doi: 10.1111/j.1399-5618.2005.00234.x

13. Regenold WT, Thapar RK, Marano C, Gavirneni S, Kondapavuluru $\mathrm{P}$ V. Increased prevalence of type 2 diabetes mellitus among psychiatric inpatients with bipolar I affective and schizoaffective disorders independent of psychotropic drug use. J Affect Disord. (2002) 70:19-26. doi: 10.1016/S0165-0327(01)00456-6

14. Fagiolini A, Chengappa KNR, Soreca I, Chang J. Bipolar disorder and the metabolic syndrome: causal factors, psychiatric outcomes and economic burden. CNS Drugs. (2008) 22:65569. doi: 10.2165/00023210-200822080-00004

15. Ruzickova M, Slaney C, Garnham J, Alda M. Clinical features of bipolar disorder with and without comorbid diabetes mellitus. Can J Psychiatry. (2003) 48:458-61. doi: 10.1177/070674370304800705

16. Bastard JP, Maachi M, Lagathu C, Kim MJ, Caron M, Vidal H, et al. Recent advances in the relationship between obesity, inflammation, and insulin resistance. Eur Cytokine Netw. (2006) 17:4-12.

17. Kaur J. A comprehensive review on metabolic syndrome. Cardiol Res Pract. (2014) 2014:943162. doi: 10.1155/2014/943162 
18. Calkin C, Van De Velde C, RuŽičková M, Slaney C, Garnham J, Hajek T, et al. Can body mass index help predict outcome in patients with bipolar disorder? Bipolar Disord. (2009) 11:650-6. doi: 10.1111/j.1399-5618.2009.00730.x

19. Bhuvaneswar CG, Baldessarini RJ, Harsh VL, Alpert JE. Adverse endocrine and metabolic effects of psychotropic drugs: selective clinical review. CNS Drugs. (2009) 23:1003-21. doi: 10.2165/11530020-000000000-00000

20. Raphael T, Parsons JP. Blood sugar studies in dementia praecox and manic-depressive insanity. Arch Neurol Psychiatry. (1921) 5:687709. doi: 10.1001/archneurpsyc. 1921.02180300052005

21. Henneman DH, Altschule MD, Goncz RM. Carbohydrate metabolism in brain disease: II. Glucose metabolism in schizophrenic, manic-depressive, and involutional psychoses. AMA Arch Intern Med. (1954) 94:40216. doi: 10.1001/archinte. 1954.00250030072008

22. Cairns K, McCarvill T, Ruzickova M, Calkin CV. Course of bipolar illness worsens after onset of insulin resistance. J Psychiatr Res. (2018) 102:347. doi: 10.1016/j.jpsychires.2018.03.006

23. Calkin CV, Alda M. Insulin resistance in bipolar disorder: relevance to routine clinical care. Bipolar Disord. (2015) 17:683-8. doi: 10.1111/bdi.12330

24. Łojko D, Owecki M, Suwalska A. Impaired glucose metabolism in bipolar patients: the role of psychiatrists in its detection and management. Int $J$ Environ Res Public Health. (2019) 16:1132. doi: 10.3390/ijerph16071132

25. Haroon E, Raison CL, Miller AH. Psychoneuroimmunology meets neuropsychopharmacology: translational implications of the impact of inflammation on behavior. Neuropsychopharmacology. (2012) 37:13762. doi: 10.1038/npp.2011.205

26. Belvederi Murri M, Prestia D, Mondelli V, Pariante C, Patti S, Olivieri B, et al. The HPA axis in bipolar disorder: systematic review and meta-analysis. Psychoneuroendocrinology. (2016) 63:327-42. doi: 10.1016/j.psyneuen.2015.10.014

27. Watson S, Gallagher P, Ritchie JC, Ferrier IN, Young AH. Hypothalamicpituitary-adrenal axis function in patients with bipolar disorder. $\mathrm{Br} J$ Psychiatry. (2004) 184:496-502. doi: 10.1192/bjp.184.6.496

28. Rosenblat JD, McIntyre RS. Bipolar disorder and immune dysfunction: epidemiological findings, proposed pathophysiology and clinical implications. Brain Sci. (2017) 7:144. doi: 10.3390/brainsci7110144

29. Goldstein BI, Kemp DE, Soczynska JK, McIntyre RS. Inflammation and the phenomenology, pathophysiology, comorbidity, and treatment of bipolar disorder: a systematic review of the literature. J Clin Psychiatry. (2009) 70:1078-90. doi: 10.4088/JCP.08r04505

30. Miller AH, Raison CL. The role of inflammation in depression: from evolutionary imperative to modern treatment target. Nat Rev Immunol. (2016) 16:22-34. doi: 10.1038/nri.2015.5

31. Cervantes P, Gelber S, Ng Ying Kin FNK, Nair VNP, Schwartz G. Circadian secretion of cortisol in bipolar disorder. J Psychiatry Neurosci. (2001) 26:411-6.

32. Rybakowski JK, Twardowska K. The dexamethasone/corticotropinreleasing hormone test in depression in bipolar and unipolar affective illness. J Psychiatr Res. (1999) 33:363-70. doi: 10.1016/S0022-3956(99) 00014-X

33. Sylvia LG, Dupuy JM, Ostacher MJ, Cowperthwait CM, Hay AC, Sachs GS, et al. Sleep disturbance in euthymic bipolar patients. J Psychopharmacol. (2012) 26:1108-12. doi: 10.1177/0269881111421973

34. Cappuccio FP, D’Elia L, Strazzullo P, Miller MA. Quantity and quality of sleep and incidence of type 2 diabetes: a systematic review and meta-analysis. Diabetes Care. (2010) 33:414-20. doi: 10.2337/dc09-1124

35. Jeremiah OJ, Cousins G, Leacy FP, Kirby BP, Ryan BK. Evaluation of the effect of insulin sensitivity-enhancing lifestyle- and dietary-related adjuncts on antidepressant treatment response: protocol for a systematic review and meta-analysis. Syst Rev. (2019) 8:62. doi: 10.1186/s13643-019-0978-8

36. Patel JP, Frey BN. Disruption in the blood-brain barrier: the missing link between brain and body inflammation in bipolar disorder? Neural Plast. (2015) 2015:708306. doi: 10.1155/2015/708306

37. Miller AH, Maletic V, Raison CL. Inflammation and its discontents: the role of cytokines in the pathophysiology of major depression. Biol Psychiatry. (2009) 65:732-41. doi: 10.1016/j.biopsych.2008.11.029

38. Modabbernia A, Taslimi S, Brietzke E, Ashrafi M. Cytokine alterations in bipolar disorder: a meta-analysis of 30 studies. Biol Psychiatry. (2013) 74:15-25. doi: 10.1016/j.biopsych.2013.01.007
39. Isgren A, Jakobsson J, Pålsson E, Ekman CJ, Johansson AGM, Sellgren C, et al. Increased cerebrospinal fluid interleukin- 8 in bipolar disorder patients associated with lithium and antipsychotic treatment. Brain Behav Immun. (2015) 43:198-204. doi: 10.1016/j.bbi.2014.10.001

40. Rao JS, Harry GJ, Rapoport SI, Kim HW. Increased excitotoxicity and neuroinflammatory markers in postmortem frontal cortex from bipolar disorder patients. Mol Psychiatry. (2010) 15:384-92. doi: 10.1038/mp.2009.47

41. Steiner J, Bielau H, Brisch R, Danos P, Ullrich O, Mawrin C, et al. Immunological aspects in the neurobiology of suicide: elevated microglial density in schizophrenia and depression is associated with suicide. J Psychiatr Res. (2008) 42:151-7. doi: 10.1016/j.jpsychires.2006.10.013

42. Bonaccorso S, Marino V, Puzella A, Pasquini M, Biondi M, Artini M, et al. Increased depressive ratings in patients with hepatitis $C$ receiving interferon- $\alpha$-based immunotherapy are related to interferon- $\alpha$-induced changes in the serotonergic system. J Clin Psychopharmacol. (2002) 22:8690. doi: 10.1097/00004714-200202000-00014

43. Bonaccorso S, Puzella A, Marino V, Pasquini M, Biondi M, Artini M, et al. Immunotherapy with interferon-alpha in patients affected by chronic hepatitis C induces an intercorrelated stimulation of the cytokine network and an increase in depressive and anxiety symptoms. Psychiatry Res. (2001) 105:45-55. doi: 10.1016/S0165-1781(01)00315-8

44. Capuron L, Gumnick JF, Musselman DL, Lawson DH, Reemsnyder $A$, Nemeroff CB, et al. Neurobehavioral effects of interferon- $\alpha$ in cancer patients: phenomenology and paroxetine responsiveness of symptom dimensions. Neuropsychopharmacology. (2002) 26:643-52. doi: 10.1016/S0893-133X(01)00407-9

45. Reichenberg A, Yirmiya R, Schuld A, Kraus T, Haack M, Morag A, et al. Cytokine-associated emotional and cognitive disturbances in humans. Arch Gen Psychiatry. (2001) 58:445-52. doi: 10.1001/archpsyc.58.5.445

46. Harrison NA, Brydon L, Walker C, Gray MA, Steptoe A, Critchley HD. Inflammation causes mood changes through alterations in subgenual cingulate activity and mesolimbic connectivity. Biol Psychiatry. (2009) 66:407-14. doi: 10.1016/j.biopsych.2009.03.015

47. Eisenberger NI, Inagaki TK, Mashal NM, Irwin MR. Inflammation and social experience: an inflammatory challenge induces feelings of social disconnection in addition to depressed mood. Brain Behav Immun. (2010) 24:558-63. doi: 10.1016/j.bbi.2009.12.009

48. Müller N. COX-2 inhibitors, aspirin, and other potential antiinflammatory treatments for psychiatric disorders. Front Psychiatry. (2019) 10:375. doi: 10.3389/fpsyt.2019.00375

49. Tyring S, Gottlieb A, Papp K, Gordon K, Leonardi C, Wang A, et al. Etanercept and clinical outcomes, fatigue, and depression in psoriasis: double-blind placebo-controlled randomised phase III trial. Lancet. (2006) 367:29-35. doi: 10.1016/S0140-6736(05)67763-X

50. Müller N, Strassnig M, Schwarz MJ, Ulmschneider M, Riedel M. COX-2 inhibitors as adjunctive therapy in schizophrenia. Exp Opin Investig Drugs. (2004) 13:1033-44. doi: 10.1517/13543784.13.8.1033

51. Mucci F, Marazziti D, Della Vecchia A, Baroni S, Morana P, Carpita B, et al. State-of-the-art: inflammatory and metabolic markers in mood disorders. Life. (2020) 10:1-23. doi: 10.3390/life10060082

52. Kim YK, Jung HG, Myint AM, Kim H, Park SH. Imbalance between proinflammatory and anti-inflammatory cytokines in bipolar disorder. J Affect Disord. (2007) 104:91-5. doi: 10.1016/j.jad.2007.02.018

53. Guimarães Barbosa I, Bauer ME, Machado-Vieira R, Teixeira AL. Review article cytokines in bipolar disorder: paving the way for neuroprogression. Neural Plast. (2014) 2014:360481. doi: 10.1155/2014/360481

54. Brietzke E, Stertz L, Fernandes BS, Kauer-Sant'Anna M, Mascarenhas M, Escosteguy Vargas A, et al. Comparison of cytokine levels in depressed, manic and euthymic patients with bipolar disorder. J Affect Disord. (2009) 116:214-7. doi: 10.1016/j.jad.2008.12.001

55. Rowland T, Perry BI, Upthegrove R, Barnes N, Chatterjee J, Gallacher D, et al. Neurotrophins, cytokines, oxidative stress mediators and mood state in bipolar disorder: Systematic review and meta-analyses. Br J Psychiatry. (2018) 213:514-25. doi: 10.1192/bjp.2018.144

56. Tsai SYM, Yang YY, Kuo CJ, Chen CC, Leu SJC. Effects of symptomatic severity on elevation of plasma soluble interleukin2 receptor in bipolar mania. J Affect Disord. (2001) 64:18593. doi: $10.1016 / \mathrm{S} 0165-0327(00) 00252-4$ 
57. Andreazza AC, Kauer-Sant'Anna M, Frey BN, Bond DJ, Kapczinski F, Young LT, et al. Oxidative stress markers in bipolar disorder: A meta-analysis. $J$ Affect Disord. (2008) 111:135-44. doi: 10.1016/j.jad.2008.04.013

58. Benedetti F, Aggio V, Pratesi ML, Greco G, Furlan R. Neuroinflammation in bipolar depression. Front Psychiatry. (2020) 11:71. doi: 10.3389/fpsyt.2020.00071

59. Sheline YI, Gado MH, Price JL. Amygdala core nuclei volumes are decreased in recurrent major depression. Neuroreport. (1998) 9:20238. doi: 10.1097/00001756-199806220-00021

60. Sheline YI, Wang PW, Gado MH, Csernansky JG, Vannier MW. Hippocampal atrophy in recurrent major depression. Proc Natl Acad Sci USA. (1996) 93:3908-13. doi: 10.1073/pnas.93.9.3908

61. Arteaga-Henríquez G, Simon MS, Burger B, Weidinger E, Wijkhuijs A, Arolt V, et al. Low-grade inflammation as a predictor of antidepressant and anti-inflammatory therapy response in MDD patients: a systematic review of the literature in combination with an analysis of experimental data collected in the EU-Moodinflame consortium. Front Psychiatry. (2019) 10:458. doi: 10.3389/fpsyt.2019.00458

62. Brunello N, Alboni S, Capone G, Benatti C, Blom JMC, Tascedda F, et al. Acetylsalicylic acid accelerates the antidepressant effect of fluoxetine in the chronic escape deficit model of depression. Int Clin Psychopharmacol. (2006) 21:219-25. doi: 10.1097/00004850-200607000-00004

63. Boufidou F, Nikolaou C, Alevizos B, Liappas IA, Christodoulou GN. Cytokine production in bipolar affective disorder patients under lithium treatment. J Affect Disord. (2004) 82:309-13. doi: 10.1016/j.jad.2004.01.007

64. Kniff E, Kupka R, Ruwhof C, Breumis M, Prens E, Nolen W, et al. Evidence that the immunopathogenic mechanism of lithium-induced psoriasis differs from that of regular psoriasis. Bipolar Disord. (2005) 7:3889. doi: 10.1111/j.1399-5618.2005.00214.x

65. Knijff EM, Nadine BM, Kupka RW, De Wit HJ, Ruwhof C, Akkerhuis GW, et al. An imbalance in the production of IL- $1 \beta$ and IL- 6 by monocytes of bipolar patients: restoration by lithium treatment. Bipolar Disord. (2007) 9:743-53. doi: 10.1111/j.1399-5618.2007.00444.x

66. Hatch JK, Scola G, Olowoyeye O, Collins JE, Andreazza AC, Moody A, et al. Inflammatory markers and brain-derived neurotrophic factor as potential bridges linking bipolar disorder and cardiovascular risk among adolescents. J Clin Psychiatry. (2017) 78:e286-93. doi: 10.4088/JCP.16m10762

67. Andela CD, Van Haalen FM, Ragnarsson O, Papakokkinou E, Johannsson G, Santos A, et al. Cushing's syndrome causes irreversible effects on the human brain: a systematic review of structural and functional magnetic resonance imaging studies. Eur J Endocrinol. (2015) 173:R114. doi: 10.1530/EJE-14-1101

68. Donath MY. Targeting inflammation in the treatment of type 2 diabetes: time to start. Nat Rev Drug Discov. (2014) 13:465-76. doi: 10.1038/ $\operatorname{nrd} 4275$

69. Calkin CV, Gardner DM, Ransom T, Alda M. The relationship between bipolar disorder and type 2 diabetes: more than just co-morbid disorders. Ann Med. (2013) 45:171-81. doi: 10.3109/07853890.2012. 687835

70. Kemp DE, Gao K, Chan PK, Ganocy SJ, Findling RL, Calabrese JR. Medical comorbidity in bipolar disorder: relationship between illnesses of the endocrine/metabolic system and treatment outcome. Bipolar Disord. (2010) 12:404-13. doi: 10.1111/j.1399-5618.2010.00823.x

71. Abbott NJ, Friedman A. Overview and introduction: the bloodbrain barrier in health and disease. Epilepsia. (2012) 53(Suppl. 6):1-6. doi: 10.1111/j.1528-1167.2012.03696.x

72. Abbott NJ, Patabendige AAK, Dolman DEM, Yusof SR, Begley DJ. Structure and function of the blood-brain barrier. Neurobiol Dis. (2010) 37:1325. doi: 10.1016/j.nbd.2009.07.030

73. Serlin Y, Shelef I, Knyazer B, Friedman A. Anatomy and physiology of the blood-brain barrier. Semin Cell Dev Biol. (2015) 38:2-6. doi: 10.1016/j.semcdb.2015.01.002

74. Pardridge WM. Blood-brain barrier carrier-mediated transport and brain metabolism of amino acids. Neurochem Res. (1998) 23:635-44.

75. Kamintsky L, Beyea SD, Fisk JD, Hashmi JA, Omisade A, Calkin C, et al. Blood-brain barrier leakage in systemic lupus erythematosus is associated with gray matter loss and cognitive impairment. Ann Rheum Dis. (2020) 26:1-6. doi: 10.1136/annrheumdis-2021-220057
76. Garg N, Smith TW. An update on immunopathogenesis, diagnosis, and treatment of multiple sclerosis. Brain Behav. (2015) 5:e00362. doi: 10.1002/brb3.362

77. Tomkins O, Shelef I, Kaizerman I, Eliushin A, Afawi Z, Misk A, et al. Bloodbrain barrier disruption in post-traumatic epilepsy. J Neurol Neurosurg Psychiatry. (2008) 79:774-7. doi: 10.1136/jnnp.2007.126425

78. Sweeney MD, Sagare AP, Zlokovic BV. Blood-brain barrier breakdown in Alzheimer's disease and other neurodegenerative disorders. Nat Rev Neurol. (2018) 14:133-50. doi: 10.1038/nrneurol.2017.188

79. Cramer SP, Simonsen H, Frederiksen JL, Rostrup E, Larsson HBW. Abnormal blood-brain barrier permeability in normal appearing white matter in multiple sclerosis investigated by MRI. NeuroImage Clin. (2014) 4:182-9. doi: 10.1016/j.nicl.2013.12.001

80. Weissberg I, Wood L, Kamintsky L, Vazquez O, Milikovsky DZ, Alexander A, et al. Albumin induces excitatory synaptogenesis through astrocytic TGF- $\beta$ /ALK5 signaling in a model of acquired epilepsy following blood-brain barrier dysfunction. Neurobiol Dis. (2015) 78:11525. doi: 10.1016/j.nbd.2015.02.029

81. Najjar S, Pearlman DM, Devinsky O, Najjar A, Zagzag D. Neurovascular unit dysfunction with blood-brain barrier hyperpermeability contributes to major depressive disorder: a review of clinical and experimental evidence. (2013) 10:142. doi: 10.1186/1742-2094-10-142

82. Pfau ML, Ménard C, Russo SJ. Inflammatory mediators in mood disorders: therapeutic opportunities. Annu Rev Pharmacol Toxicol. (2018) 58:41139. doi: 10.1146/annurev-pharmtox-010617-052823

83. Gudmundsson P, Skoog I, Waern M, Blennow K, Palsson S, Rosengren $\mathrm{L}$, et al. The relationship between cerebrospinal fluid biomarkers and depression in elderly women. Am J Geriatr Psychiatry. (2007) 15:8328. doi: 10.1097/JGP.0b013e3180547091

84. Hultman K, Strickland S, Norris EH. The APOE $\varepsilon 4 / \varepsilon 4$ genotype potentiates vascular fibrin(ogen) deposition in amyloid-laden vessels in the brains of Alzheimer's disease patients. J Cereb Blood Flow Metab. (2013) 33:12518. doi: $10.1038 /$ jcbfm. 2013.76

85. Rajkowska G, Stockmeier C. Astrocyte pathology in major depressive disorder: insights from human postmortem brain tissue. Curr Drug Targets. (2013) 14:1225-36. doi: 10.2174/13894501113149990156

86. Weissberg I, Veksler R, Kamintsky L, Saar-Ashkenazy R, Milikovsky DZ, Shelef I, et al. Imaging blood-brain barrier dysfunction in football players. JAMA Neurol. (2014) 71:1453-5. doi: 10.1001/jamaneurol.2014.2682

87. Serlin Y, Ofer J, Ben-Arie G, Veksler R, Ifergane G, Shelef I, et al. Blood-brain barrier leakage: a new biomarker in transient ischemic attacks. Stroke. (2019) 50:1266-9. doi: 10.1161/STROKEAHA.119.025247

88. Varatharaj A, Galea I. The blood-brain barrier in systemic inflammation. Brain Behav Immunity. (2017) 60:1-12. doi: 10.1016/j.bbi.2016.03.010

89. Friedman A, Kaufer D, Heinemann U. Blood-brain barrier breakdowninducing astrocytic transformation: novel targets for the prevention of epilepsy. Epilepsy Res. (2009) 85:142-9. doi: 10.1016/j.eplepsyres.2009.03.005

90. Cacheaux LP, Ivens S, David Y, Lakhter AJ, Bar-Klein G, Shapira $M$, et al. Transcriptome profiling reveals TGF- $\beta$ signaling involvement in epileptogenesis. J Neurosci. (2009) 29:8927-35. doi: 10.1523/JNEUROSCI.0430-09.2009

91. Menard C, Pfau ML, Hodes GE, Kana V, Wang VX, Bouchard S, et al. Social stress induces neurovascular pathology promoting depression. Nat Neurosci. (2017) doi: 10.1038/s41593-017-0010-3

92. Greene C, Hanley N, Campbell M. Blood-brain barrier associated tight junction disruption is a hallmark feature of major psychiatric disorders. Transl Psychiatry. (2020) 10:373. doi: 10.1038/s41398-02001054-3

93. Konradi C, Sillivan SE, Clay HB. Mitochondria, oligodendrocytes and inflammation in bipolar disorder: evidence from transcriptome studies points to intriguing parallels with multiple sclerosis. Neurobiol Dis. (2012) 45:37-47. doi: 10.1016/j.nbd.2011.01.025

94. Van Dyken P, Lacoste B. Impact of metabolic syndrome on neuroinflammation and the blood-brain barrier. Front Neurosci. (2018) 12:930. doi: 10.3389/fnins.2018.00930

95. Bogush M, Heldt NA, Persidsky Y. Blood brain barrier injury in diabetes: unrecognized effects on brain and cognition. J Neuroimmune Pharmacol. (2017) 12:593-601. doi: 10.1007/s11481-017-9752-7 
96. Mooradian AD. Dyslipidemia in type 2 diabetes mellitus. Nat Clin Pract Endocrinol Metab. (2009) 5:150-9. doi: 10.1038/ncpendmet1066

97. Kellar D, Craft S. Brain insulin resistance in Alzheimer's disease and related disorders: mechanisms and therapeutic approaches. Lancet Neurol. (2020) 19:758-66. doi: 10.1016/S1474-4422(20)30231-3

98. Kahn BB, Alquier T, Carling D, Hardie DG. AMP-activated protein kinase: ancient energy gauge provides clues to modern understanding of metabolism. Cell Metab. (2005) 1:15-25. doi: 10.1016/j.cmet.2004.12.003

99. Janus A, Szahidewicz-Krupska E, Mazur G, Doroszko A. Insulin resistance and endothelial dysfunction constitute a common therapeutic target in cardiometabolic disorders. Mediators Inflamm. (2016) 2016:3634948. doi: 10.1155/2016/3634948

100. David Y, Cacheaux LP, Ivens S, Lapilover E, Heinemann U, Kaufer D, et al. Astrocytic dysfunction in epileptogenesis: consequence of altered potassium and glutamate homeostasis? J Neurosci. (2009) 29:1058899. doi: 10.1523/JNEUROSCI.2323-09.2009

101. Vazana U, Veksler R, Pell GS, Prager O, Fassler M, Chassidim Y, et al. Glutamate-mediated blood-brain barrier opening: implications for neuroprotection and drug delivery. J Neurosci. (2016) 36:772739. doi: 10.1523/JNEUROSCI.0587-16.2016

102. Lapin IP, Oxenkrug GF. Intensification of the central serotoninergic processes as a possible determinant of the thymoleptic effect. Lancet. (1969) 293:132-6. doi: 10.1016/S0140-6736(69)91140-4

103. Chiarugi A, Meli E, Moroni F. Similarities and differences in the neuronal death processes activated by $3 \mathrm{OH}$-kynurenine and quinolinic acid. J Neurochem. (2001) 77:1310-8. doi: 10.1046/j.1471-4159.2001. 00335.x

104. Etain B, Dumaine A, Bellivier F, Pagan C, Francelle L, Goubran-botros H, et al. Genetic and functional abnormalities of the melatonin biosynthesis pathway in patients with bipolar disorder. Hum Mol Genet. (2012) 21:40307. doi: $10.1093 / \mathrm{hmg} / \mathrm{dds} 227$

105. Bar-Klein G, Cacheaux LP, Kamintsky L, Prager O, Weissberg I, Schoknecht $\mathrm{K}$, et al. Losartan prevents acquired epilepsy via TGF- $\beta$ signaling suppression. Ann Neurol. (2014) 75:864-75. doi: 10.1002/ana.24147

106. Senatorov VV, Friedman AR, Milikovsky DZ, Ofer J, SaarAshkenazy R, Charbash A, et al. Blood-brain barrier dysfunction in aging induces hyperactivation of TGF $\beta$ signaling and chronic yet reversible neural dysfunction. Sci Transl Med. (2019) 11:eaaw8283. doi: 10.1126/scitranslmed.aaw8283

107. Boese AC, Lee JP, Hamblin MH. Neurovascular protection by peroxisome proliferator-activated receptor $\alpha$ in ischemic stroke. Exp Neurol. (2020) 331:113323. doi: 10.1016/j.expneurol.2020.113323

108. Danielson M, Reinsfelt B, Westerlind A, Zetterberg H, Blennow K, Ricksten SE. Effects of methylprednisolone on blood-brain barrier and cerebral inflammation in cardiac surgery - a randomized trial. J Neuroinflammation. (2018) 15:283. doi: 10.1186/s12974-018-1318-y

109. Liu Y, Tang G, Li Y, Wang Y, Chen X, Gu X, et al. Metformin attenuates blood-brain barrier disruption in mice following middle cerebral artery occlusion. J Neuroinflammation. (2014) 11:177. doi: 10.1186/s12974-014-0177-4

110. Mudgal J, Nampoothiri M, Basu Mallik S, Kinra M, Hall S, Grant $\mathrm{G}$, et al. Possible involvement of metformin in downregulation of neuroinflammation and associated behavioural changes in mice. Inflammopharmacology. (2019) 27:941-8. doi: 10.1007/s10787-019-00638-w

111. Lashen $H$. Role of metformin in the management of polycystic ovary syndrome. Ther Adv Endocrinol Metab. (2010) 1:11728. doi: $10.1177 / 2042018810380215$

112. Abdelgadir E, Ali R, Rashid F, Bashier A. Effect of metformin on different non-diabetes related conditions, a special focus on malignant conditions: review of literature. J Clin Med Res. (2017) 9:388-95. doi: 10.14740/jocmr2922e
113. Jia Y, Ma Z, Liu X, Zhou W, He S, Xu X, et al. Metformin prevents DMHinduced colorectal cancer in diabetic rats by reversing the warburg effect. Cancer Med. (2015) 4:1730-41. doi: 10.1002/cam4.521

114. Lakhan SE, Kirchgessner A, Tepper D, Leonard A. Matrix metalloproteinases and blood-brain barrier disruption in acute ischemic stroke. Front Neurol. (2013) 4 APR:32. doi: 10.3389/fneur.2013.00032

115. Benešová $Y$, Vǎko A, Novotná H, Litzman J, Štourač P, Beránek M, et al. Matrix metalloproteinase- 9 and matrix metalloproteinase-2 as biomarkers of various courses in multiple sclerosis. Mult Scler. (2009) 15:31622. doi: $10.1177 / 1352458509348421$

116. Konstantino Y, Pfizer ST, Nguyen TT, Wolk R, Aiello RJ, Terra SG, et al. Potential implications of matrix metalloproteinase- 9 in assessment and treatment of coronary artery disease. Biomarkers. (2009) 14:11829. doi: 10.1080/13547500902765140

117. Olson FJ, Schmidt C, Gummesson A, Sigurdardottir V, Hulthe J, Wiklund $\mathrm{O}$, et al. Circulating matrix metalloproteinase 9 levels in relation to sampling methods, femoral and carotid atherosclerosis. J Intern Med. (2008) 263:62635. doi: 10.1111/j.1365-2796.2008.01927.x

118. Dhingra R, Pencina MJ, Schrader P, Wang TJ, Levy D, Pencina K, et al. Relations of matrix remodeling biomarkers to blood pressure progression and incidence of hypertension in the community. Circulation. (2009) 119:1101-7. doi: 10.1161/CIRCULATIONAHA.108.821769

119. Rybakowski JK, Remlinger-Molenda A, Czech-Kucharska A, Wojcicka M, Michalak M, Losy J. Increased serum matrix metalloproteinase-9 (MMP-9) levels in young patients during bipolar depression. J Affect Disord. (2013) 146:286-9. doi: 10.1016/j.jad.2012.07.019

120. Metformin Inhibits Tumor Cell Migration via Down-regulation of MMP9 in Tamoxifen-resistant Breast Cancer Cells. Available online at: http://ar. iiarjournals.org/content/34/8/4127.short

121. Kang HS, Cho HC, Lee JH, Oh GT, Koo SH, Park BH, et al. Metformin stimulates IGFBP-2 gene expression through PPARalpha in diabetic states. Sci Rep. (2016) 6:1-13. doi: 10.1038/srep23665

122. Zhao Y, Wei X, Song J, Zhang M, Huang T, Qin J. Peroxisome proliferator-activated receptor $\gamma$ agonist rosiglitazone protects bloodbrain barrier integrity following diffuse axonal injury by decreasing the levels of inflammatory mediators through a caveolin-1-dependent pathway. Inflammation. (2019) 42:841-56. doi: 10.1007/s10753-018-0940-2

123. Mandrekar-Colucci S, Sauerbeck A, Popovich PG, McTigue DM. PPAR agonists as therapeutics for CNS trauma and neurological diseases. ASN Neuro. (2013) 5:AN20130030. doi: 10.1042/AN20130030

124. Matthews DR, Hosker JP, Rudenski AS, Naylor BA, Treacher DF, Turner RC. Homeostasis model assessment: insulin resistance and $\beta$-cell function from fasting plasma glucose and insulin concentrations in man. Diabetologia. (1985) 28:412-9. doi: 10.1007/BF00280883

125. Esteghamati A, Ashraf H, Khalilzadeh O, Zandieh A, Nakhjavani M, Rashidi A, et al. Optimal cut-off of homeostasis model assessment of insulin resistance (HOMA-IR) for the diagnosis of metabolic syndrome: Third national surveillance of risk factors of non-communicable diseases in Iran (SuRFNCD-2007). Nutr Metab. (2010) 7:26. doi: 10.1186/1743-7075-7-26

Conflict of Interest: The authors declare that the research was conducted in the absence of any commercial or financial relationships that could be construed as a potential conflict of interest.

Copyright (C) 2021 Calkin, McClelland, Cairns, Kamintsky and Friedman. This is an open-access article distributed under the terms of the Creative Commons Attribution License (CC BY). The use, distribution or reproduction in other forums is permitted, provided the original author(s) and the copyright owner(s) are credited and that the original publication in this journal is cited, in accordance with accepted academic practice. No use, distribution or reproduction is permitted which does not comply with these terms. 